\title{
INFORMATIONEN und BERICHTE
}

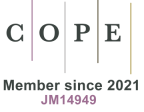

https://doi.org/10.18778/2196-8403.2021.12

\begin{abstract}
„Shared Heritage - gemeinsames Erbe. Kulturelle Interferenzräume im östlichen Europa als Sujet der Gegenwartsliteratur". Internationale Tagung des Bundesinstituts für Kultur und Geschichte der Deutschen im östlichen Europa (BKGE) im Literaturhaus Berlin und online, 19. - 21.11.2020
\end{abstract}

Die von Lesungen und Gesprächen mit Autor*innen aus dem östlichen Europa begleitete Tagung wurde von der Bundesbeauftragten für Kultur und Medien im Rahmen der deutschen EU-Ratspräsidentschaft gefördert und vom BKGE veranstaltet.

Die Tagung verfolgte das Ziel, anhand von postmemorialen Texten der Gegenwart die literarische Auseinandersetzung mit dem gemeinsamen bzw. geteilten Erbe in kulturellen Interferenzräumen zu beleuchten und das Potenzial des Konzeptes exemplarisch anhand des östlichen Europas und seinen häufig multiethnischen Regionen mit deutschsprachigen Bevölkerungsanteilen $\mathrm{zu}$ untersuchen. Ausgangspunkt war die Beobachtung, dass sich zahlreiche Schriftsteller*innen mit dem (traumatischen) Erbe in osteuropäischen Interferenzräumen beschäftigen und sich diesem zumeist aus dem distanzierteren Blickwinkel der Nachfolgegenerationen nähern. $\mathrm{Zu}$ den zentralen Anliegen der Tagung gehörten die Frage nach den Übertragungsmöglichkeiten des aus der Denkmalpflege stammenden Shared Heritage-Begriffs von materiellen auf immaterielle Kulturgüter und die damit verbundene Diskussion des literaturwissenschaftlichen Erkenntniswerts dieses Konzepts. Besonderes Untersuchungsinteresse galt dem erinnerungskulturellen und -politischen Potential literarischer Erinnerungen aus der Distanz der Kinder- bzw. Enkel*innengeneration sowie den narrativen Formen, Strategien und Genres dieser postmemorialen Literatur.

Auftakt der Tagung bildeten die Ansprache der Beauftragten der Bundesregierung für Kultur und Medien Prof. Dr. MONIKA GRÜTTERS, der Keynote-Vortrag Deutschpolnische Interferenzräume. Zur Entropie der nationalen Kultur von Prof. Dr. JOANNA JABŁKOWSKA (Łódź) sowie ein von Dr. SILKE BEHL moderiertes Podiumsgespräch mit den Autor*innen JosePH ZODERER, CATALIN DORIAN FLORESCU und SABRINA JANESCH. Verantwortlich für das Konzept und die Durchführung der Tagung war Dr. SiLKE PASEWALCK (BKGE).

Zur Einleitung des ersten Panels Zur Poetologie literarischer Shared Heritage-Texte erläuterte und problematisierte SILKE PASEWALCK (Oldenburg) das aus der Kunst- und Denkmalpflege stammende Konzept des Shared Heritage und stellte dessen literaturwissenschaftliche Anschlussfähigkeit zur Diskussion. Um das Potenzial des ideellen Konzepts eines geteilten kulturellen Erbes herauszustellen, bezog sie sich insbesondere auf den polnischen Kunsthistoriker 
Andrzej Tomaszewski, der mit dem Konzept sowohl ein gemeinsames Interesse als auch eine gemeinsame Verantwortung verbindet und daraus den Auftrag der Erhaltung und Pflege des Kulturerbes ableitet. Gleichzeitig sensibilisierte PASEWALCK für die Gefahren der Instrumentalisierung des Konzepts und reflektierte über den Unterschied von shared heritage und sharing heritage. Literarischen Texten, die den Umgang mit einem z.T. schwierigen kulturellen Erbe verhandeln, schrieb PASEWALCK eine Brückenund Reflexionsfunktion zu, da diese das Gemeinsame und das Trennende inklusiv behandelten und eine kollektive Teilhabe am Erbe ermöglichten.

Im ersten Vortrag , Auf der Suche nach der (verlorenen) Mitte - Literarische Spurensuche im östlichen Europa ging HANSCHRISTIAN TREPTE (Leipzig) der geografischen, politisch-ideologischen, kulturhistorischen wie auch identitätsstiftenden Frage nach der Bestimmung Mittel- bzw. Zentraleuropas nach. Angesichts aktueller Renationalisierungs- und Abgrenzungsbestrebungen sowie der damit einhergehenden Desillusionierung von Visionen eines geeinten Europas betonte TREPTE das Potenzial der Literatur im Verständigungsprozess über ein gemeinsames, jedoch auch traumatisiertes europäisches Erbe.

KATARZYNA ŚliwiŃSKa (Poznań) befasste sich in ihrem Vortrag mit problematischen Shared Things? Zum Potential des Unheimlichen in der polnischen Gegenwartsliteratur über die ehemals deutschen Regionen des heutigen Polen. Sie zeigte auf, wie die traumatische Vergangenheit in den materiellen, präsentischen Spuren der vertriebenen Deutschen als gespenstische Heimsuchung vergegenwärtigt werde und inwiefern dabei auch diffuse Ängste und Schuldgefühle der Polen im Zusammenhang mit der gewaltsamen Verdrängung der deutschen Bevölkerung zum Ausdruck gebracht würden. Die Annäherung an die Sphäre des Alltäglichen und Privaten der Deutschen öffne einen Diskursraum, in dem die kollektiven Imaginationen des Deutschen revidiert würden.

In ihrem Vortrag Zwischen Echo und Palimpsest wand sich GUDRUN HEIDEMANN (Łódź) dem Umgang mit problematischen Erbstücken in drei Zeugnissen polnischer Gegenwartsliteratur zu. Das dialektische Aufheben von ehemals deutschen Gegenständen in Olga Tokarczuks Roman Taghaus, Nachthaus (1998), der parodistische Umgang mit prominenten Prätexten in Tomasz Różyckis subversiver Heimatdichtung Zwölf Stationen (2004) sowie die konkrete Sichtbarmachung eines traumatischen Familienerbes und seiner transgenerationalen Weitergabe in Agata Baras Comic Der Garten (2012) deutete HeIDEMANN dabei als verschiedene Strategien literarischer Erbteilung.

EszTER PROPSZT (Szeged) interpretierte im Rahmen ihres Beitrags das Phänomen des geteilten Erbes in ungarndeutscher Literatur aus raumsemiotischer Perspektive. Mithilfe von Juri M. Lotmans Konzept der Raumsemantik und der Semiosphäre untersuchte sie Prozesse der Aneignung und Teilung von Erbe im als Emailwechsel angelegten Roman Schlafen werden wir später (2017) von Zsuzsa Bánk und zeigte auf, dass in einem erweiterten Raum der dialogischen Teilhabe, Mitwirkung und Mitverantwortung am Erbteilungsprozess letztlich auch die Leserschaft miteinbezogen werde.

SABINE KYORA (Oldenburg) diskutierte unter Rückgriff auf den Interferenzbegriff des Philosophen Michel Serres die literarischen Konstruktionsmöglichkeiten kultureller Interferenzräume und machte die Idee von nicht hierarchisch geordneten Netzwerkstrukturen für die Konzeptualisierung von Erbteilungsprozessen fruchtbar. In den 
deutschsprachigen Romanen Herkunft (2019) von Saša Stanišić, Vielleicht Esther (2014) von Katja Petrowskaja und Die Listensammlerin (2013) von Lena Gorelik identifizierte sie die Verschmelzung kultureller Räume von Figuren, Erzähler*innen und Leser*innen, die Schichtung und Pluralisierung von Orten, Zeitebenen und Herkünften sowie die Schaffung eines privaten Interferenzraumes auf dem Papier als unterschiedliche Strategien einer literarischen Konfliktforschung und Angebote für ein Shared Heritage.

Im letzten Vortrag des ersten Panels analysierte JACQUELINE GUTJAHR (Göttingen) die Inszenierung von Erinnerung sowie die poetische Hervorbringung des slowenischen Erinnerungsraums karantanien in Maja Haderlaps Gedichtzyklus langer transit (2014). Dabei zeigte sie auf, dass der Text nicht nur im Modus des Erinnerns verfasst sei, sondern zugleich die Mechanismen von Erinnerung und kollektiver Gedächtnisbildung im Sinne einer erinnerungskulturellen Performance vorführe, was eine Reflexion über die Vermittlung von Geschichtsbildern und Prozessen der kulturellen Selbstauslegung anstoße.

Im Zentrum der von BEATE STÖRTKUHL und SiLKe PASEWALCK moderierten Diskussionen des ersten Panels standen sowohl die kritische Reflexion des Shared HeritageBegriffs als auch die Frage nach der Bedeutung kultureller Interferenzräume und ihrer mangelnden Eindeutigkeit für Identitätsbildung, Erinnerungskultur und Gedenken. Besonderes Interesse galt der Diskussion des geteilten Erbes als Konfliktforschung im Sinne Sigrid Weigels sowie der Betrachtung von literarischen Netzwerkstrategien nicht als Aufhebung, sondern vielmehr als Sichtbarmachung von Konflikten.

Im zweiten Panel der Tagung lag der Schwerpunkt auf kulturellen Interferenzräumen im östlichen Europa als Sujet der deutschsprachigen Gegenwartsliteratur, welche die Referent*innen am Beispiel von Schlesien, Galizien, Böhmen, Siebenbürgen/Banat und Ostbelgien erkundeten.

ALEKSANDRA BURDZIEJ (Toruń) beschäftigte sich in ihrem Beitrag Geteiltes Trauma, gemeinsames Erbe mit der literarischen Überwindung der Unversöhnlichkeit deutschpolnischer Erinnerungen an Flucht und Vertreibung. Am Beispiel von Sabrina Janeschs Katzenberge (2010) und Ulrike Draesners Sieben Sprünge vom Rand der Welt (2014) fragte sie nach den narrativen Möglichkeiten, die von Reinhart Koselleck postulierte ,gebrochene Erinnerung“ in ein gemeinsam geteiltes Erbe zu überführen.

ERIK SCHILLING (München) widmete seinen Vortrag ebenfalls Ulrike Draesners Roman Sieben Sprünge vom Rand der Welt (2014) und untersuchte den multiperspektivischen Raum kultureller Interferenzen vor der Folie aktueller Positionen von Geschichts- und Kulturtheorie. Für die Reflexion der Frage danach, wie Geschichte und Gedächtnis sich konstituieren, verglich er Hans Ulrich Gumbrechts Konzept der breiten Gegenwart mit der kritischen Erweiterung durch Aleida Assmanns Theorie der Kulturalisierung der Zeit und stellte darauf aufbauend die Pluralität der Wahrnehmung von Geschichte in Draesners Roman heraus. In ihrem Vortrag zum Thema Das jüdische Erbe Mittel- und Osteuropas vermitteln zeichnete Alina MolisAK (Warschau) zwei wichtige Strömungen der polnischen Gegenwartsliteratur in Hinblick auf die Erinnerung an die polnisch-jüdische Vergangenheit nach. Die erste, sog. schwarze Strömung erscheine dabei als Abrechnung mit der Kriegsvergangenheit und Bewusstmachung der tabuisierten und oft verschwiegenen polnischen Verhaltensweisen und Haltungen gegenüber den Jüdinnen und Juden, wohingegen im Rahmen der zweiten, sog. 
inklusiven Strömung die polnisch-jüdische Kontaktzone im Sinne von Mary Louise Pratt rekonstruiert und im Zuge dessen an die jüdische Erbschaft im heutigen Polen erinnert werde.

Michaela Nowotnick (Berlin) untersuchte in ihrem Beitrag die literarische Verarbeitung der individuellen und gesellschaftlichen Folgen des rumäniendeutschen Exodus in zeitgenössischer deutschsprachiger Prosaliteratur. Am Beispiel von Nadine Schneiders Drei Kilometer (2019) und Thomas Perles Wir gingen, weil alle gingen (2018) zeigte sie zum einen die neue Beschäftigung der jungen rumäniendeutschen Autor*innengeneration mit der Abwanderung und dem Aussterben der deutschsprachigen Gemeinschaft in Rumänien und las zum anderen Frieda Schullers bislang unveröffentlichten Roman Doctor Draculescu als letztes Denkmal für die rumäniendeutsche Prägung Osteuropas.

In seinem Beitrag Regionale Verflechtungen und gemeinsames Erbe unternahm ARVI SEPP (Brüssel/Antwerpen) einen Vergleich transkultureller Erinnerungsräume in der ostbelgischen und rumäniendeutschen $\mathrm{Ge}-$ genwartsliteratur. Dafür zog er zwei Beispiele deutschsprachiger Minderheitenliteratur aus radikal unterschiedlichen geschichtlichen, politischen und kulturellen Kontexten heran: Freddy Derwahls Bosch in Belgien (2006) und Balthasar Waitzs Krähensommer und andere Geschichten aus dem Hinterland (2011). Aus postnationaler Perspektive setzte SEPP diese miteinander in Verbindung, um zu zeigen, wie der regionalen Spezifik, aber auch der Vergleichbarkeit der jeweiligen kulturellen Interferenzräume mit ihren Beziehungen zur deutschen und zugleich ostbelgischen bzw. rumänischen Kultur Ausdruck verliehen wird.

Im letzten Vortrag dieses Panels erkundete ANNE Hultsch (Wien) Nordböhmen als gemeinsamen deutsch-tschechischen Erzählraum und beobachtete als Gemeinsamkeit der ausgewählten transkulturellen Erinnerungstexte ihre zumindest thematische Multimedialität: die Frottage bei Radek Friedrich, die Malerei bei Václav Vokolek und der Film bei Veronika Bendová. Die Wertschätzung für das gemeinsame deutsch-tschechische Erbe drückt sich HULTSCH zufolge darin aus, dass man es sich produktiv durch Überführung in ein anderes Medium aneigne, seine Geschichte nachverfolge und sich gleichzeitig selbst in die Geschichte einschreibe. Schwerpunkte der von JOANNA JABŁKOWSKA und HANS-CHRISTIAN TREPTE moderierten Diskussionen im Rahmen des zweiten Panels bildeten sowohl die Erinnerungsund Gedächtnisproblematik als auch die Krise des Konzepts von historischer Wahrheit, Archivwissen und Augenzeugenschaft sowie die daraus resultierende Unsicherheit bei der Geschichtsschreibung und -rekonstruktion. Bereichert wurde die Diskussion über traumatische Erbprozesse insbesondere durch Reflexionen über den Einfluss der Neurowissenschaften auf den Traumadiskurs, der hierdurch in Richtung Empathie umgelenkt werde und eine tendenzielle Abkehr vom psychotherapeutischklinischen Diskurs ankündige.

Im dritten und letzten Panel Das Verhältnis zum Erbe in der Gegenwartsliteratur setzte sich zuerst CSONGOR LÖRINCZ (Berlin) in seinem Beitrag zu Texten der rumäniendeutschen Autorin Herta Müller und des ungarischsprachigen rumänischen Autors Ádám Bodor mit dem Phänomen des Erbes und den das Erbe kennzeichnenden Prozessen der Desemiotisierung nach Renate Lachmann auseinander. Ausgehend von Jacques Derridas These, dass ein Erbe nur als Geheimnis erfahrbar sei, beleuchtete LÖRINCZ insbesondere das im Rahmen dieser Tagung wiederkehrende Mo- 
tiv des Gespenstischen und Unheimlichen vor allem auch auf der Ebene der Sprache. In ihrem Vortrag Zur Deterritorialisierung von Gedächtnisorten stellte SABINE EGGER (Limerick) die These auf, dass die spezifische Ästhetik der Bewegung in Katja Petrowskajas Vielleicht Esther (2014) und Anna Burns' Milkman (2018) sowohl einen transterritorialen Erinnerungsraum als auch einen sich ständig verändernden Zugehörigkeitsraum schaffe. Anknüpfend an Überlegungen von Gilles Deleuze und Félix Guattari untersuchte sie die Rhizomstruktur des postmemorialen Erfahrungsraums in Petrowskajas Text, der durch die Deterritorialisierung von Versuchen des Ichs, sich über seine Familiengeschichte zu verorten, entstehe. Vor diesem Hintergrund skizzierte EGGER die im Kontrast zu den genealogischen Verbindungen stehenden affiliativen Verbindungslinien zu den Opfern der Geschichte und beleuchtet den durch literarische Fluchtlinien entstehenden multidirektionalen Erinnerungsraum im Sinne Michael Rothbergs.

Im letzten Vortrag setzte sich SILKE PASEWALCK (Oldenburg) mit dem Konzept von Familie und Erbe in Gusel Jachinas historischem Roman Wolgakinder (2019) auseinander und deutete das Annehmen eines kulturellen Erbes durch nicht familiär Betroffene als Akt der Patenschaft bzw. Adoption. Ihre These entwickelte sie entlang der im Roman entworfenen komplexen und ambivalenten Vaterschaft, die sich nicht auf Biologie oder Tradition, sondern vielmehr auf Fürsorge und Weitergabe gründe. Im Kontrast dazu las sie das Motiv des Verstummens und der Verweigerung der Sprachweitergabe als Ausdruck des Schweigens der traumatisierten Elterngeneration und der damit verbundenen transgenerationalen Weitergabe des Traumas. Die Tatsache, dass sich eine russische Autorin der Geschichte der Wolga- deutschen annimmt, ohne selbst im Sinne der familiären Herkunft zu dieser Gruppe zu gehören, ließ PASEWALCK fragen, ob Wolgakinder damit einen Spezialfall oder gar eine Steigerung aktueller Shared Heritage-Texte darstellt.

In der von ARVI SEPP moderierten Diskussion zu den Vorträgen in diesem letzten Panel standen vor allem Fragen der (kulturellen) Übersetzung, des Phänomens der latenten und manifesten Mehrsprachigkeit sowie die Räumlichkeit der Texte und die damit verbundene Beziehung zwischen Bewegung, Wahrnehmung und Narrativität im Vordergrund.

Im Resümee der Tagung wurde der Boom bzw. Trend der postmemorialen Literatur angesichts der Wahrscheinlichkeit, dass eine Vielzahl an Texten nur in Bezug auf vorgängige kulturelle und politische Erwartungen produziert wurde, kritisch reflektiert und zugleich nach politischen Motivationen des postmemorialen Schreibens gefragt. Im weiteren Verlauf diskutierten die Referent*innen über die gesellschaftlichen Funktionen des literarischen Erinnerungsdiskurses und damit über die Funktion von Literatur als Erinnerungsspeicher, das Anschreiben gegen das Vergessen, aber auch gegen Falschdeutungen sowie das Konzept einer Geschichtsschreibung von unten statt einer Erinnerungspolitik von oben. Der literarische Gegendiskurs zur nationalen Geschichtsschreibung wurde dabei als Diskurs der Verunsicherung von kollektiver Erinnerung und Identität gefasst. Angesichts der Tatsache, dass sich insbesondere die Enkelinnengeneration der Verantwortung des (traumatischen) Erbes stellt, wurden auch generationen- und genderspezifische Aspekte dieser Literatur in den Blick genommen. Abschließend diskutierten die Tagungsteilnehmer*innen, inwiefern kulturelles Erbe jenseits persönlicher bzw. 
familiärer Betroffenheit weniger als $\mathrm{Pa}$ tenschaft oder Adoption als vielmehr Miterbschaft konzipiert werden kann, um den Gefahren der Reproduktion von Erbfolge- hierarchien und Deutungshoheiten entgegenzuwirken.

Caren Bea Henze, Freiburg

\section{„Kunst.Politik.Moral“. Interdisziplinäres Online-Symposium, 01.-04.05.2021}

Die Veranstaltung wurde vom Interuniversitären Forschungsverbund (IFV) Elfriede Jelinek und Elfriede Jelinek-Forschungszentrum in Kooperation mit dem Tanzquartier Wien und dem Österreichischen Kulturforum Warschau organisiert. Weitere Kooperationspartner waren die Central European University Vienna, das Institut für Germanistik und die Abteilung für deutschsprachige Medien und österreichische Kultur der Universität Lodz, das Institut für Germanistik der Universität Warschau, das Institut für Kulturwissenschaften der Universität Bydgoszcz, die Österreich-Bibliothek Warschau, die Theaterakademie Warschau, das Theater in der Josefstadt, das sirene Operntheater und das TR (Teatr Rozmaitości) Warschau. Obwohl das Symposium pandemiebedingt online abgehalten wurde, dienten zwei konkrete Orte als Anhalts- und Ausgangspunkte für die Vorträge, Gespräche und künstlerischen Beiträge. An den ersten beiden Tagen bildete das Tanzquartier Wien mit Programmpunkten vor Ort sowie online diesen Fixpunkt, an den beiden weiteren Tagen das Österreichische Kulturforum Warschau. Die gesamte Veranstaltung wurde simultan gedolmetscht und dadurch sowohl auf Deutsch als auch auf Polnisch zugänglich gemacht.

Die Teilnehmer:innen befassten sich mit Zusammenhängen von Kunst, Politik und Moral ausgehend von Elfriede Jelineks Haltung und Position als politische Künstlerin sowie mit ihrem künstlerischen An- spruch, außerdem mit Jelinek als Autorin, die mit ihrem Schreiben immer politisch und gesellschaftlich eingreifen und etwas bewirken will und dafür oftmals öffentlich angegriffen und skandalisiert wurde und wird. Zur Debatte standen überdies Fragen nach Möglichkeiten des Ausdrucks politischen Engagements und umgekehrt Fragen nach dem Schweigen und der Abwesenheit im politischen Diskurs. Zugleich wurden grundsätzliche Themen staatlicher bzw. politischer Repression, Skandalisierung und öffentlicher Diffamierung im internationalen Vergleich angesprochen. Dem Symposium lag ferner das Streben zugrunde, Wissenschaft und Kunst zu verbinden.

\section{Tag 1: Politisch(es) Schreiben: Provo- kation, Resignation, Engagement}

Pia Janke (Wien) und Andrea Heinz (Wien) eröffneten das Symposium mit einem Überblick über die Themen und über das Projekt Kunst und Politik des IFV Jelinek, welches sich mit grundsätzlichen Aspekten politischer Ästhetik befasst. Die Tagesmoderation übernahm SABRINA WeINZETTL (Wien).

Der erste Vortrag Zur Kunst politischen Schreibens: Provokation ade von BRIGITTE JIRKU (Valencia) setzte sich mit der physischen Präsenz des Autorinnenkörpers und politischer Performanz in seiner Abwesenheit am konkreten Beispiel Elfriede Jelineks auseinander. Nach einem kurzen Überblick über Jelineks künstlerisches (und gleichzeitig politisches) Engagement bis zu ihrem 
endgültigen Rückzug 2004 im Zuge der Verleihung des Nobelpreises für Literatur befasste sich die Vortragende mit den darauf folgenden Strategien des Nicht-Präsenten der Autorin. Dabei finde der Rückzug auch im Text und in der Schreibstrategie seinen Niederschlag, etwa durch das Mittel des Chors, der das Autorinnen-Ich in den Hintergrund treten lässt. Genauso spiele das Internet als eine Art Schutzschild in Raum und Zeit eine Rolle, Bild und Schrift als Archiv stünden an der Stelle Jelineks - woraus sich auch die Frage nach dem moralischen Anspruch der Autorin ergebe. Mit Verweis auf die Auswirkungen der Pandemie auf die physische Präsenz des Körpers und unter Bezugnahme auf Jelineks Engagement plädierte BRIGITTE JIRKU zum Abschluss für ein Neudenken der Frage, wer für wen spricht, besonders unter Berücksichtigung des (be)lebbaren Raumes, den es ohne diesen Körper gar nicht gäbe.

In der folgenden von ANDREA HEINZ moderierten Gesprächsrunde „Ich habe es jetzt lange genug versucht..." Aufbruch oder Resignation? diskutierten SILKe FELBER (Wien), ANITA MAYER-HIRZBERGER (Wien) und der Wiener Autor DORON RABINOVICI über zwei Pole, zwischen welchen Jelinek hin- und herpendle: zwischen dem Glauben an die Kraft der Kunst, dass es also möglich wäre, in den öffentlichen Diskurs (verändernd) eingreifen zu können, und einem sarkastischen Eingestehen der Vergeblichkeit dieses Anspruchs. Dabei ging es auch um weitere Felder bzw. Fragen, etwa wie bzw. was die gegenwärtige Situation politischer Kunst sei, wo das Politische in neuen Formen der Öffentlichkeit Platz finde, was sich verändert habe seit Jelineks Anfängen und wieso sich heute niemand mehr auf jene Art, wie Jelinek dies tat und auch immer noch tut, öffentlich äußere und mit der Gegenwart be- schäftige. Zentral war in der Debatte das Problem der Vereinnahmung durch das Kapital (oder durch die Besitzenden). Jede Selbstpositionierung müsse mit dieser Vereinnahmung umgehen, da diese ohne eigenes Mittun geschehen könne - und damit sei auch Jelineks Rückzug möglicherweise ein Versuch, dieses ökonomisch/kapitalistisch-durchflochtene Spiel „nicht mehr mitzuspielen" (SILKE FELBER).

Im Sinne der angestrebten Verbindung von Wissenschaft und Kunst präsentierten danach Doris Uhlich (Wien) und Studierende der Universität Wien sowie der MUK (Musik und Kunst Privatuniversität der Stadt Wien) via Videokonferenz Ergebnisse aus einem gemeinsamen Workshop. Ausgehend von Passagen aus Jelineks Theaterstück Am Königsweg (2017) setzten sie sich mit dem durch die Pandemie plötzlich begrenzt erscheinenden Umraum auseinander. In einer kurzen Einleitung betonte NiKolaus Selimov (Wien) bezugnehmend auf das Online-Format des Workshops, dass es keine Berührungen und keine körperlichen Begegnungen in einem gemeinsamen Handlungs- und Aktionsraum gegeben habe und DoRIS UHLICH daher den Umgebungsraum mit performativen Handlungsanweisungen für die Körperaußengrenze, die Haut, erspür-, erfühl- und erfahrbar gemacht habe. So berichteten auch die Studierenden von einer intensiven, neuartigen Auseinandersetzung mit dem scheinbar gewohnten (Wohn-)Raum und tauschten sich gemeinsam mit DORIS UhLICH über diese Erfahrungen aus.

Den letzten wissenschaftlichen Programmpunkt des ersten Tages bildete eine von CHRISTIAN SCHENKERMAYR (Wien) moderierte Online-Gesprächsrunde mit dem Titel Zwischen Postmoderne und Populismus. Perspektiven politischer Ästhetik. RoSEMARIE BRUCHER (Wien), NATASCHA STROBL 
(Wien) und DoRIS UhLICH diskutierten über Positionen und Haltungen, also über den moralischen Anspruch politischer Kunst und politischer Künstler:innen sowie über Perspektiven politischer Ästhetik. Jelinek und ihr Werk sowie die Positionierung der Künstlerin angesichts zunehmender rechter und rechtspopulistischer Tendenzen machten den Ausgangspunkt des Gesprächs aus. Besprochen wurden außerdem Fragen des künstlerischen Umgangs mit rechter, rechtsextremer und rechtspopulistischer Politik und Ästhetik auch jenseits von Jelinek etwa an Hand des Beispiels der Burschenschaft Hysteria, das NATASCHA STROBL einbrachte. In Anlehnung an den Workshop kamen auch Fragen danach auf, wie der Körper politisch sein könne und wie der politische Körper auf Rechtsextremismus und rechtsextreme Ideologien, die auf Eindeutigkeit und klaren Dichotomien beruhten, reagieren kann. Zum Abschluss des Gesprächs wurde nach möglichen Formen und Kanälen der Intervention gefragt: nach den Bubbles, in welchen sich politische Kunst bewegt und wie sie über diese hinaus hör-, seh- und fühlbar werden und damit in den Diskurs eintreten bzw. an diesem teilhaben könne.

Zum Abschluss des ersten Tages gaben der Komponist DiRK D“ASE (Wien) und die Wiener Autorin Miroslava Svolikova sowie die Studierenden des MUK JULIA MikusCh (Schauspiel), JunghyUN OH und EMma FraUENHOLZ (Schlagwerk) via Videogespräch Prozesseinsicht in die musikalisch-theatralische Performance Europa flieht. Der Beitrag gestaltete sich als eine Art Werkstattgespräch, in dem Autorin, Komponist und die teilhabenden Studierenden vom Arbeitsprozess berichteten. Ausgehend von einem Ausschnitt von MiroslaVA SVOLIKOVAS Theatertext Europa flieht nach Europa befasste sich DIRK D“ASE bezugnehmend auf die aktuellen Flucht- bewegungen in Richtung Europa und den lebensbedrohlichen bis tödlichen Überfahrten durchs Mittelmeer mit ihrem Text, der sich, so die Autorin, mit der Geschichte und der Mythologie Europas als von Neuanfängen und Brüchen geprägt und durchzogen auseinandersetzt.

\section{Tag 2: Rechnitz (Der Würgengel) in Öster- reich und Polen}

Den Rahmen des zweiten Tages bildete Elfriede Jelineks Stück Rechnitz (Der Würgeengel) aus dem Jahr 2008. Am Beginn stand eine Gesprächsrunde mit PIA JANKE, MONIKA MEISTER (Wien) und BARBARA NowOTNY (Wien), die sich mit dem Stück im Allgemeinen und einer konkreten Aufführung des Theaters in der Josefstadt auseinandersetzte und die anderen Programmpunkte für die Dauer des Abends als eine Art roter Faden begleitete. Dabei wurde über den Theatertext, dessen Entstehung und dessen historische Einordnung gesprochen. In Anlehnung an den ersten Tag wurde zudem über die Frage des politischen und moralischen Anspruchs Jelineks und über ihr Verdienst in der Erinnerungsarbeit diskutiert. Die Theaterwissenschaftlerin MONIKA MEISTER betonte dabei das öffentliche Moment des Politischen wie auch des Theaters als Ort der Auseinandersetzung des Publikums mit seiner bzw. mit der Geschichte. BARBARA NowOTNY, Dramaturgin der Produktion des Theaters in der Josefstadt, aus welcher ein Ausschnitt voraufgeführt wurde, sprach die große Motivvielfalt des Textes an, die sich auch diesem Ausschnitt zeige. Während der Gesprächsrunde wurden Videobotschaften bezugnehmend auf die Relevanz von Jelineks Stück eingeschoben: OSKar Deutsch, Präsident der Israelitischen Kultusgemeinde Wien, betonte die enorme Bedeutung der Erinnerung an die historischen Geschehnisse in Rechnitz für Österreich und die gesamte Welt. Die His- 
torikerin HeIDEMARIE UhL (Wien) sprach die Tabuisierung dieser im Osten Österreichs zu Kriegsende verübten Gräueltaten in der Nachkriegszeit an und strich die Kraft hervor, die ein Theaterstück habe, das Schweigen zu brechen und lange an die Geschehnisse zu erinnern. Die Literaturwissenschaftlerin und Literaturkritikerin DANIELA STRIGL (Wien) äußerte sich zu der äußerst vielschichtigen Abbildung der Verworrenheit und Totalität eines kollektiven gesellschaftlichen Versagens in Jelineks ,nicht-theatralischem“ Theatertext.

Nach einer Fortsetzung der oben erwähnten Gesprächsrunde wurden Ausschnitte aus der Aufführung von Jelineks Rechnitz (Der Würgeengel) mit der Schauspielerin SONA MaCDonald, inszeniert von ANNA BERGMANN und produziert vom Theater in der Josefstadt, vor Ort im Tanzquartier Wien, wo auch die Diskussion stattfand, gezeigt.

Im darauffolgenden, von JENS KASTNER (Wien) moderierten Online-Gespräch mit dem Titel Kunst als Tagespolitik? Ästhetik und Aktualität diskutierten die Wiener Schriftstellerin Olga Flor und DAVID WeBERMAN von der Central European University (Budapest/Wien) über Möglichkeiten der Verbindung von Kunst (bzw. im engeren Sinn von Literatur) und Politik und darüber, ob diese Verbindungsversuche nicht von Vornherein zum Scheitern verurteilt wären. DAVID WEBERMAN sah zwischen (Tages-) Politik und Kunst wenig Widerspruch und wenig Spannung, für ihn eröffneten beide Begriffe sehr weite Felder mit mannigfaltigen Verbindungslinien. Dahingehend wurde dann auch die Aktualität und das Ephemere des Politischen und das Universale oder Langlebige der Kunst (das ,Für-Immer-Gültige“ der Kunst oder der Anspruch der Kunst darauf) im Gegensatz dazu diskutiert. OLGA FLOR sprach in dieser Hinsicht davon, dass Kunst, die zu ihrer Epoche als gut gelte war, auch heute (und in Zukunft) gut sein müsse, da sie etwas eingefangen habe - da sie also etwas Epochenspezifisches verdichtet habe. In der danach fortgesetzten Debatte innerhalb der den Tag rahmenden Gesprächsrunde wurde zuerst die Vielstimmigkeit und die Musikalität des Rechnitz-Textes besprochen, bevor anknüpfend an das vorhergegangene Online-Gespräch die Aktualität von Jelineks Stück fokussiert wurde. Jelinek zeige in ihrem Text Strukturen von Macht, Machtmissbrauch und Gewalt auf, die bis heute produktiv seien und mache dadurch einen neuen Blick auf das Jetzt sowie gleichzeitig auf die Antike (bzw. die antiken Texte) möglich, so das Ergebnis. Darauf folgte ein weiteres online abgehaltenes Gespräch, welches zweisprachig geführt und gedolmetscht wurde. Aus der künstlerischen Praxis heraus diskutierten auf Polnisch der Dramaturg, Autor und Regisseur ŁUKASZ CHOTKOWSKI und die Theaterregisseurin KATARZYNA KALWAT sowie auf Deutsch von einem wissenschaftlichen Blickpunkt her OLIVER MARCHART (Wien) und STEFAN SCHMIDL (Wien) unter dem Titel Kunst und Moral; moderiert wurde das Gespräch von ANDREA HEINZ (Wien) und ARTUR PEŁKA (Łódź). In diesem interessant besetzten Gespräch wurden Fragen nach dem (möglicherweise antipodischen) Verhältnis und den Zusammenhängen von Kunst und Moral besprochen, es wurde nach dem Anspruch der Autonomie der Kunst auf der einen und dem moralischen Anspruch auf der anderen Seite gefragt, danach, ob und/oder inwiefern sich diese widersprechen, sich gegenseitig aushebeln - und nach einer oder der Grenze von Moral. Oliver MARCHART etablierte gleich zu Beginn die Notwendigkeit einer historischen Perspektive auf unsere Begriffe von Kunst und Moral, aber auch auf die Politik als dritte Kategorie in diesem Dreieck. STEFAn SCHMIDL wies auf die bis heute 
wirksame kunstreligiöse Dogmatik des 19. Jahrhunderts hin, von der wir uns frei machen müssten, da sie die Kunstschaffenden gleichzeitig immer als Moralschaffende sehe. KATARZYNA KALWAT betonte, dass eine Kohärenz der persönlichen Haltung mit den Themenbereichen der Beschäftigung gegeben sein müsse, dass also ein:e Künstler:in, die:r sich in der eigenen Arbeit mit emanzipatorischen Fragen auseinandersetzt, selbst eine solche Haltung vertreten müsse. Daran knüpfte LUKASZ CHOTKOwski an, der sich direkt auf Elfriede Jelinek und darauf bezog, wie sie die verlogene Weise zeige, in der Moral von Politiker:innen ausgenutzt werde: je nachdem, wozu die Moral eben gerade gebraucht werde. Des Weiteren wurde über Fragen der politischen Kunst, der Politik in der Kunst und der Kunst in der Politik gesprochen und eine gemeinsame Debatte darüber geführt, was eine ,nationale Kunst" sei bzw. welches Verhältnis zwischen Nation und Kunst bestehe. Außerdem wurde die Rezeption Jelineks in Polen im Vergleich zur Rezeption in Österreich in den Fokus genommen. Die Schauspielerin SONA MacDonald beschrieb dann zum Abschluss der Gesprächsrunde im Tanzquartier, wie sie an Jelineks Text heranging. Sie berichtete von einer Art Scheu, die sie aus Respekt vor der Autorin empfand. Sobald sie aber ,im Text' gewesen sei, habe sie sich von dessen Musikalität und der fast unendlich scheinenden Vielschichtigkeit der Worte geradezu beschenkt gefühlt. Ähnliches richtete dann ANNA BERGMANN, die das Jelinek-Stück im Theater in der Josefstadt inszenierte, in einer Videobotschaft aus, in der sie von der Inszenierung und der Arbeit am Stück und von der Herangehensweise an den Text berichtete.

Nach einer kurzen Einleitung von MONIKA Muskata, Autorin der Adaption und Übersetzerin von Rechnitz ins Polnische, die über das Konzept der Adaption, des Stücks und auch konkret über den gezeigten Ausschnitt sprach, wurden als letzter Programmpunkt des zweiten Tages Szenen aus Wojtek Blecharz' Rechnitz. Opera (Anioł Zagłady) gezeigt, einer Produktion des TR Warszawa, die von KATARZYNA KALWAT inszeniert wurde.

\section{Tag 3: Möglichkeiten des Protests}

Der dritte Tag des Symposiums fand ausschließlich online statt, konkreter und analoger Ausgangspunkt war dennoch das Österreichische Kulturforum Warschau, was der Leiter des Österreichischen Kulturforums, RUPERT WEINMANN, bei und mit seiner VideoBegrüßung zu Beginn des Programms unterstrich. Mit der Tagesmoderation war ARTUR PeŁKa betraut.

Thema dieses Tages war politischer Protest mittels Kunst und in der Kunst im internationalen Vergleich, die Frage des Standpunktes, von welchem aus gesprochen und geschrieben wird, und mögliche Ausformungen des künstlerischen Protests. AGNIESZKA JEZIERSKA-WIŚNIEWSKA (Warschau) machte mit ihrem Video-Vortrag Jelineks ästhetischer Protest im Namen der Schweigenden, gegen die politischen Schreihälse den Anfang. Sie setzte sich mit dem „Standort im Abseits" auseinander, über den Jelinek in ihrer Nobelpreisrede 2004 gesprochen hatte, und zeigte, wie dieser als lohnender Beobachtungsstandort dienen könne. Allerdings sei die:r Künstler:in im Abseits den Angriffen aus dem Zentrum ausgesetzt. Anschließend erläuterte sie zwei Dimensionen, die Jelinek in ihren Texten verbinde und gegen welche sie protestiere: jene der Verharmlosung und jene des Vergessens. Außerdem thematisierte sie Jelineks Strategien, den politischen Diskurs und die Phrasen der Herrschenden der Gegenwart und der jüngeren Vergangenheit nebeneinander $\mathrm{zu}$ stellen und gegenseitig zu entlarven. Zum Abschluss ging AgNiESZKA JEZIERSKA-WIŚNIEWSKA 
im Gespräch mit ARTUR PeŁKA den Spezifika der Jelinek-Rezeption in Polen nach und stellte einen Vergleich kritischer Literatur und Literat:innen in Polen und in Österreich an. Im nächsten online abgehaltenen Gespräch Staatskunst oder Nestbeschmutzung? Politische Kunst im internationalen Vergleich mit ZuZANA Augustovì (Pilsen), KAROLINE EXNER (Wien), ANTON PELINKA (Innsbruck) und WERONIKA SZCZAWIŃSKA (Warschau), das von MONIKa SZCZEPANIAK (Bydgoszcz) moderiert wurde, ging es um das politischästhetische Engagement Jelineks und um ihre Texte in einer politischen Atmosphäre, die von Kunstfeindlichkeit und der Diffamierung politischer Künstler:innen geprägt war und teilweise heute noch ist. Ziel des Gesprächs war es, im Kontext des internationalen Vormarsches rechtsradikaler Parteien und der Verbreitung ihrer Diskurse über politische Kunst zu diskutieren, aber auch und damit verbunden über die Frage von Skandalen und Skandalisierung in der Kunst und in der Politik nachzudenken. Gleich zu Beginn der Runde unterstrich Anton Pelinka, dass Politik und Kunst zwei gesellschaftliche Bereiche seien, die voneinander nicht zu trennen wären, vielmehr würden sich beide überlappen und besäßen mannigfaltige Schnittstellen sowie Schnittmengen. ZuZANA Augustovà brachte einen vergleichenden Blick in die Diskussion ein, indem sie die Strategien österreichischer und tschechischer politischer Kunst, gegen die Mächtigen aufzubegehren, kontrastierte. Eine dieser Strategien sprach KAROLINE EXNER an. Sie führte aus, dass Jelinek in der Sprache das Groteske und Komische einer Realität offenlege, die eine übersteigerte Parodie ihrer selbst zu sein scheine, wo Täter:innen mit einer ins Komödiantische kippenden übertriebenen Vehemenz sich selbst zu Opfern machten. WERONIKA SZCZAWIŃSKA nahm die gegenwärtige Situation in Polen in den
Fokus und äußerte sich zur Problematik und den Chancen der zeitgenössischen polnischen Theateröffentlichkeit. Sie betonte die Notwendigkeit des Wiederaufbaus von Netzwerken und Diskursen, welche die dringenden Themen der Zeit angehen und jene Foren schaffen könnten, in welchen Diskussionen ohne Tabus möglich wären.

Die letzte Videokonferenz des Tages mit dem Titel Eine Frau darfkein Werk haben widmete sich bezugnehmend auf dieses Zitat Jelineks von 1997 der Frage nach dem Ort oder dem Nicht-Ort von Frauen in der Kunst und dem Problem der (Un-)Sichtbarkeit von Künstlerinnen vor dem Hintergrund ihrer Positionen, Rollen und Aufgaben im transnationalen Vergleich. Es diskutierten Claudia Breger (New York) und Asako FukUOKa (Tokyo), moderiert wurde die Diskussion von Stefan Krammer (Wien). Claudia Breger bemerkte in Hinsicht auf die titelgebende Frage, dass diese zum einen überhaupt nicht aktuell sei, wenn es etwa darum gehe, dass Frauen weit einfacher publizieren könnten als noch vor 25 Jahren. Zum anderen gäbe es jedoch immer noch männlich dominierte Felder im Literaturbetrieb, die weit starrer und veränderungsresistenter seien. Ähnliches beobachtet ASAKO FUKUOKA in Japan, wo Frauen heute in der Literatur und in sozialen Medien eindeutig präsenter seien als vor einigen Jahren. Dennoch erlaubten verschiedene soziale Phänomene es Frauen in Japan nicht, ,ein Werk zu haben“, wofür sie neben anderem einen rechtspopulistischen Backlash und eine gesamtgesellschaftliche neoliberale Tendenz verantwortlich zeichnete.

Den abschließenden Beitrag des Tages bildete die 15-minütige Online-Performance Die Schutzbefohlenen - Atlantis, die die beiden Studierenden der Theaterakademie Warschau AgATA KosZuLIŃSKA und MATEUSZ KORSAK vorführten. 
Tag 4: Aufführung, Skandal und Rezeption Jelineks in Polen

Das leitende Thema des letzten Tages war die Rezeption von Elfriede Jelineks Texten und Stücken in Polen. Am Beginn des Programms stand eine Begrüßung durch MACIEJ A. SOBORCZYK, Leiter der Österreich-Bibliothek Warschau. MONIKA SZCZEPANIAK, die die Tagesmoderation übernahm, betonte zum Einstieg, dass die Auseinandersetzung mit Elfriede Jelinek in Polen so intensiv wie in kaum einem anderen (nicht-deutschsprachigen) europäischen Land sei.

Bevor das Tagesthema in Angriff genommen wurde, präsentierten TATJANA GRIESSLER und SABRINA WeINZETTL, beide wissenschaftliche Mitarbeiterinnen beim IFV Jelinek, das Digital Humanities Projekt Jelinek Online. In dessen Rahmen wird Jelineks Gesamtwerk und dessen Rezeption bibliographisch erschlossen, kommentiert und in Form eines Online-Portals bereitgestellt. Jelineks heterogenes Werk stellt an die literaturwissenschaftliche Dokumentation besondere Herausforderungen, die durch die im Projekt genutzten Methoden der Digital Humanities adäquat bewältigt werden könnten. In ihrem Online-Vortrag Zwischen Kunst und Politik. Der Theaterskandal um die polnische Inszenierung von Jelineks Prinzessinnendramen im Teatr Polski beschäftigte sich dann ANNA MaJKIEwICZ (Częstochowa) mit der bemerkenswerten Aufführung von Jelineks Prinzessinendramen (Der Tod und das Mädchen I-V; 2003) in der Breslauer Inszenierung von Ewelina Marciniak von 2015. Sie sprach sowohl über Idee, Konzept, Bühnenbild und die durchgängige Hervorhebung des Körpers in der Inszenierung als auch über den Skandal, den diese auslöste, der politische Auswirkungen nach sich zog und in rechten und rechtsextremen Protesten gegen die Aufführung des Stücks gipfelte. ANNA MAJKIEWICZ bekräftigte außerdem, dass Elfriede Jelinek vor allem durch Die Kla- vierspielerin (1983) und der immer noch virulenten Auseinandersetzung mit diesem Roman als Lese- wie auch als Theatertext einen festen Platz in der polnischen Literaturund Kulturlandschaft einnehme.

Zum Abschluss des Symposiums beleuchteten BoŻENA CHOŁUJ (Frankfurt a.d. Oder), AnNa MajKIEWICZ und Monika MuskąA in einer von SABRINA WEINZETTL moderierten Online-Gesprächsrunde mit dem Titel „Solche Stücke [...] wollen wir nicht auf unseren Bühnen sehen!" die Rezeption Jelineks in Polen im Allgemeinen. Obwohl sich der polemische Ausspruch, ein JelinekZitat von 1997, auf Österreich bezog, könne dieser, so SABRINA WEINZETTL einleitend, auch für Polen gelten. Das Gespräch drehte sich um Skandale und Reaktionen im Zusammenhang mit Aufführungen Jelineks in Polen und um die Frage, warum gerade ihre Texte so provozieren. Weiters wurde über öffentliche und mediale Sanktionierungsmaßnahmen, Reaktionen auf unbequeme Aufführungen und nicht zuletzt über die Aktualität von Jelineks Texten diskutiert. Bożena CHOŁUJ hob hervor, dass die Radikalität Jelineks ein Grund sei, warum sie so stark rezipiert werde. Ihre politische Wirkung sei eine heftige, da sie inhaltlich wie formal radikal schreibe, wodurch sie eine:n nicht in Ruhe lasse und sogar jene damit reize, die mit dieser Theaterästhetik nichts anfangen könnten. Als weitere Ursache für die ausgeprägte Rezeption der Autorin machte ANNA MAJKIEWICZ die Tatsache aus, dass Jelinek seit der Verleihung des Nobelpreises für Literatur bis heute in Polen (und überhaupt im nicht-deutschsprachigen Kontext) vorrangig als Feministin rezipiert werde, da diese Perspektive universell und nicht kulturspezifisch sei. MoniKa MusKaŁa griff diese häufig monolithische Deutung des Werkes Jelineks auf und berichtete über die spezielle Herausforderung der Übersetzungsarbeit 
bei Jelinek, die sich aus den vielen Anspielungen in ihren Texten und den eingewobenen Verflechtungen der Vergangenheit mit der Gegenwart ergebe. Sogleich unterstrich sie die Relevanz der Übersetzung von Jelineks Werk. Denn die Auflehnung und der Widerstand gegen das Vergessen sei in ihren Texten zentral. Und eben dieser Widerstand sei für das zeitgenössische Polen und seine Politik von großer Bedeutung.

Das hybrid durchgeführte Symposium zeigte, dass fruchtbare Diskussionen und gemeinsames Nachdenken und Debattieren - wenn notwendig - auch ohne physisches Zusammensein möglich sind. Gleichzeitig wurde in Anlehnung an Jelineks künstlerische Praxis und an das von ihr (auch) als Protestform gewählte Abwesend-Sein offensichtlich, dass die physische Präsenz und der präsente Körper eine wichtige Rolle in der Form des politischen Ausdrucks spielen - und sei es in der selbstgewählten Negation dieser Präsenz. Aber auch viele andere Themenkomplexe rund um die Möglichkeit des politischen Protests, des Widerstands und des Engagements wurden aufgerollt und betrachtet. Die Aktualität dieser Themen und der Texte Jelineks in Anbetracht sich politisch nach rechts verschiebender und in
Form wie in Inhalt extremer werdender diskursiver Auseinandersetzungen in nahezu allen gesellschaftlichen Bereichen wurde offensichtlich. Augenscheinlich wurde auch die Relevanz des Jelinek'schen Werkes für die gegenwärtige polnische Politik und Gesellschaft sowie für das polnische Theater. Nicht zuletzt verdeutlichte das Symposium eindrucksvoll, wie wichtig und ergiebig die Verbindung österreichischer, polnischer und weiterer internationaler Wissenschaftsstandorte ist.

\section{Literatur}

JeLINEK, Elfriede (1983): Die Klavierspielerin. Hamburg.

JeLINEK, ELFRIEDE (2003): Der Todund das Mädchen I-V. Prinzessinendramen. Berlin. JeLINEK, Elfriede (2008): Rechnitz (Der Würgeengel). In: JELINEK, ElFRIEDE: Drei Theaterstücke. Die Kontrakte des Kaufmanns; Rechnitz (Der Würgeengel); Über Tiere. Hamburg, 53-205.

JeLINEK, ELFRIEDE (2014): Die Schutzbefohlenen. In: Theater heute 07/2014:3-19. JELINEK, ELFRIEDE (2017): Am Königsweg. In: Theater heute 12/2017 (Beilage).

Christian Poik, Łódź 